\title{
40
}

\section{Mutation versus All-uses: An Empirical Evaluation of Cost, Strength and Effectiveness}

\author{
W. Eric Wong ${ }^{a}$, Aditya P. Mathur ${ }^{b}$ and José C. Maldonado ${ }^{c}$ \\ ${ }^{a}$ Department of Software Technology, Hughes Network Systems, Germantown, MD 20876. \\ ${ }^{b}$ Department of Computer Sciences, Purdue University, W. Lafayette, IN 47907. \\ ${ }^{c}$ Department of Computer Science and Statistics, University of São Paulo, 13560-970, \\ São Carlos, Brazil.
}

\begin{abstract}
Although mutation has been empirically found to be effective in detecting faults, it often imposes unacceptable demands on computing and human resources because of the large number of mutants that need to be compiled and executed on one or more test cases. We designed a case study to explore two alternatives of mutation to reduce its cost without significantly deteriorating its strength and effectiveness. The alternatives are (1) constrained abs/ror mutation which examines only the abs and ror mutants and ignores the others, and (2) randomly selected $10 \%$ mutation which examines only $10 \%$ of the randomly selected mutants of each mutation operator in Mothra. Data collected during experimentation have shown that both alternatives provide a significant cost reduction in terms of the number of test cases required to satisfy an adequacy criterion and the number of mutants to be examined. Such gain is accompanied by a small strength loss in the ability to distinguish non-equivalent mutants and cover feasible all-uses. In terms of decreasing order of fault detection effectiveness the ranking is mutation, constrained $a b s /$ ror mutation, all-uses, and randomly selected $10 \%$ mutation. These data suggest that examining only a small percentage of the mutants may be a useful heuristic for evaluating and constructing test sets in practice.
\end{abstract}

Keyword Codes: D.2.5; D.2.8

Keywords: Software Testing, Cost, Strength, Effectiveness, Mutation, All-uses

\section{Introduction}

Although mutation testing has been empirically found to be effective in detecting faults[3, 7], it suffers from a problem of performance. The complexity of mutation is $O\left(n^{2}\right)$, where $n$ is the number of variable references in the program. The number of mutants generated can be prohibitively large even for programs that are less than $100 \mathrm{~K}$ lines of code. These mutants have to be compiled, executed, and often examined by the tester to determine equivalence, which makes mutation testing too expensive to be used in practice. Different algorithms were proposed to reduce the time to execute all mutants on a parallel [9] and a sequential [11] machine. These algorithms suffer either a conversion between different operating environments or extra memory requirement to store the program state, which limits their usability. As a significant portion of the computational cost of mutation testing is incurred in executing mutants against test cases, this cost can be reduced significantly by reducing the number of mutants to be 
examined. We, therefore, propose the randomly selected $10 \%$ mutation and the constrained abs/ror mutation criteria described below. The major difference between our approach and the algorithms discussed above is that those algorithms do not reduce the number of mutants to be examined; it is the time to execute the mutants that is targeted for reduction whereas both of them are reduced in our approach.

Cost, difficulty of satisfaction, and fault detection effectiveness [12] are three meaningful bases against which test adequacy criteria should be compared. Cost refers to the work necessary to satisfy it. Difficulty of satisfaction is also known as the subsumption relationship [5] and strength [10]. Fault detection effectiveness refers to the fault detection capability of a criterion. We conducted experiments to explore the two alternatives of mutation described above with respect to the all-uses and mutation criteria in terms of these three factors. Data collected during experimentation will help us to answer the questions: Will reducing the number of mutants to be examined be a cost-effective alternative to the mutation testing? Will examining only a small percentage of the mutants be a useful heuristic for evaluating and constructing test sets in practice?

The remainder of this paper is organized as follows. Section 2 provides a brief overview of mutation and data flow based adequacy criteria used in the remainder of this paper. In Section 3, we explain how we compared the cost, strength, and effectiveness of various test adequacy criteria. Our experimental methodology is described in detail in Section 4. Data collected from experiments and its analysis appear in Section 5. Our conclusions appear in Section 6 .

\section{All-uses and mutation based test adequacy criteria}

In this section we provide an overview of all-uses and mutation based test adequacy criteria by presenting only the information required for an understanding of the rest of the paper. More details can be found in $[5,7]$. Let $P$ denote the program under test with $\mathcal{D}$ as its input domain. Test cases are selected from the input domain. A test set $T \subseteq \mathcal{D}$ consists of one or more test cases.

\subsection{All-uses criterion}

Let $s_{i}$ and $s_{j}, 1 \leq i, j \leq n$ be two statements in $P$ at which variable $x$ is defined, $d_{i}(x)$, and used, $u_{j}(x)$, respectively. The pair $\left(d_{i}(x), u_{j}(x)\right)$ is a def-use pair. A def-use pair is a $p$-use or a $c$-use pair depending on whether $s_{j}$ is a predicate or not, respectively. We say that any such pair is feasible if there exists a test case $t \in D$ such that the execution of $P$ on $t$ causes control to transfer from $s_{i}$ to $s_{j}$, traversing one or more statements, without defining $x$ at any statement other than at $s_{i}$. Such a path is also considered a definition clear path with respect to $x$. A test case $t \in T$ covers a c-use pair $\left(d_{i}(x), u_{j}(x)\right)$ if the execution of $P$ on $t$ causes the execution of a definition clear path with respect to $x$ from $s_{i}$ to $s_{j}$. A p-use pair $\left(d_{i}(x), u_{j}(x)\right)$ is considered covered if a definition clear path with respect to $x$ from $s_{i}$ to $s_{j}$ to $s_{k}$ is executed for each successor $s_{k}$ of $s_{j}$. We refer to the set of all c-uses and p-uses as all-uses [5]. A test set $T$ may be evaluated against the all-uses criterion by computing the ratio of the total number of all-uses covered to the total number of feasible all-uses. A ratio of unity implies that $T$ is adequate with respect to the all-uses criterion.

\subsection{Mutation criterion}

Let $m$ be a syntactically correct program obtained by making a syntactic change in $P ; m$ is known as a mutant of $P$. Let $r$ be a rule according to which $P$ is changed; $r$ is known as a mutant operator. There could potentially be an infinite number of mutant operators. To keep mutation testing practical, the set of mutant operators is kept small and consists of simple mutant operators [4]. The application of a set of mutant operators $M O$ to $P$ results in a set 
of mutants $m_{1}, m_{2}, \ldots, m_{n}, n \geq 0$. Mutant $m_{i}$ is considered equivalent to $P$ if for all $t \in \mathcal{D}$, $P(t)=m_{i}(t)$. When executed against a test case $t$, mutant $m_{i}$ is considered distinguished from $P$ if $P(t) \neq m_{i}(t)$. Unless distinguished, a non-equivalent mutant is considered live. One can obtain a variety of mutation based criteria by varying $M O$. Hereafter, we refer to the mutation based criterion with respect to the set of all 22 mutant operators in Mothra [4] as the mutation criterion. A test set $T$ may be evaluated against the mutation criterion by executing each mutant against elements of $T$. The ratio of the number of mutants distinguished to the number of nonequivalent mutants is the mutation score of $T$ for $P . T$ is considered adequate with respect to the mutation criterion if the mutation score is unity.

One mechanism for reducing the cost of mutation testing is to examine a small percentage, say $10 \%$, of randomly selected mutants of each mutant type and ignore the others. Earlier empirical investigations by Acree [1], Sayward, and Budd [3] have shown that even with test cases generated using only $10 \%$ of the mutants, no more than $1 \%$ of the non-equivalent mutants were overlooked. In this paper we present results not only to verify this aspect but also to investigate the cost and effectiveness of the randomly selected $10 \%$ mutation. Hereafter, we refer to such alternate mutation as $10 \%$ mutation.

Another mechanism for reducing the cost of mutation testing is constrained mutation. Using constrained mutation, one examines only a few specified types of mutants and ignores the others. For example, the constrained abs/ror mutation studied in this paper examines only the abs and ror mutants. The $a b s$ mutant operator generates mutants by replacing each use of $x$ by $a b s(x),-a b s(x)$, and $z p u s h(x)$ wherever possible. The terms, $a b s(x)$ and $-a b s(x)$ represent the absolute value and the negative absolute value of $x$, respectively. A mutant containing $z p u s h(x)$ is considered distinguished if a test case causes the value of $x$ to be zero when execution reaches the mutated expression. When applied to a program containing an assignment statement $z:=x+1$, the $a b s$ operator generates six mutants obtained by replacing this assignment by $z:=a b s(x)+1, z:=-a b s(x)+1, z:=z p u s h(x)+1, z:=a b s(x+1), z:=-a b s(x+1)$, and $z:=z p u s h(x+1)$. The ror mutant operator generates mutants by replacing each relational operator by other relational operators. In addition, it also replaces each condition consisting of at least one relational operator by the boolean constants true and false. For example, when applied to a program containing a predicate "if $(x=0)$ then", this mutant operator generates the following seven conditions: "if $(x<0)$ then", "if $(x \leq 0)$ then", "if $(x \neq 0)$ then", "if $(x>0)$ then", "if ( $x \geq 0)$ then", "if (true) then", and "if (false) then". A detailed rationale of such selection can be found in [14]. Hereafter, we refer to the constrained mutation using abs and ror mutation operators as abs/ror mutation.

\section{Comparison methodology}

The cost of the alternative mutation testing is measured by two metrics. One is the number of test cases required to satisfy it [10]. The other is the number of mutants to be examined [14]. We use Equations (1) and (2) to compute the cost reduction in terms of the number of test cases required with respect to the mutation or all-uses adequacy criteria, respectively, and the cost reduction in terms of the number of mutants examined with respect to the mutation adequacy criterion.

$\left(1-\frac{\text { average size of test sets adequate with respect to alternate mutation }}{\text { average size of mutation (or all-uses) adequate test sets }}\right) * 100 \%$

$$
\left.-\frac{\text { total number of mutants examined when using alternate mutation }}{\text { total number of mutants examined in mutation }}\right) * 100 \%
$$

Two measures were selected for the strength of the alternative mutation testing. In one, the strength is measured in terms of the mutation scores by Equation (3). In the other, the strength is measured in terms of the all-uses scores by Equation (4).

$\frac{\text { number of mutants distinguished by a test set }}{\text { total number of non-equivalent mutants }} * 100 \%$ 
number of all-uses covered by a test set

total number of feasible all-uses $* 100 \%$

Given a program $P$, a specification $S$, and a test set $T$ adequate with respect to a criterion $C$, $T$ is said to be able to expose faults in $P$ if there exists a test case $t \in T$ which makes $P$ behave differently from $S$. As long as $T$ detects at least one fault in $P, T$ is counted as a fault-revealing test set. Equation (5), defined below, is used to compute the percentage of $C$ fault-revealing adequate test sets.

number of $C$ adequate test sets which expose at least one fault number of $C$ adequate test sets generated $* 100 \%$

\section{Experimental methodology}

\subsection{Subject programs}

For cost and strength comparison, we used a suite of the four programs described below. Faults discovered while preparing the programs were removed. This was done as the goal of our experiments was to investigate which one of the adequacy criteria is more expensive and difficult to satisfy and not to investigate their fault detection capability. A source listing of each of these programs and removed faults can be found in [13].

- FIND: This program takes two inputs, an array $a$ and an index $f$, and permutes the elements of $a$ so that elements to the right of position $f$ are greater than or equal to $a[f]$ and elements to the left of position $f$ are less than or equal to $a[f]$.

- STRMAT1 and STRMAT2: Each program takes a text and a pattern of zero or more characters. If the pattern appears in the text then the position of the first occurrence of the pattern in the text is returned, otherwise a zero is returned.

- TEXTFMT: This program takes a text as input and formats it.

For effectiveness comparison, we used another suite of four programs with naturally occurring faults described below. A source listing of each of these programs and their faults can also be found in [13].

- SORT: This program takes an array and sorts it in descending order.

- STRMAT3: This program is similar to STRMAT1 and STRMAT2 with faults not removed.

- POSITION: This program takes two inputs, an array $a$ and a value max, and sums the elements of $a$ until the sum meets or exceeds max. If such an element exists, its position is returned, otherwise, a zero is returned.

- STAT: This program takes an array $a$ and computes its sum, minimum, and maximum.

\subsection{Experiments \& testing tools}

We generated mutants in our experiments using all 22 mutant operators in Mothra except the goto. Two testing tools, Mothra and ATAC [8] were used. Mothra is a mutation testing tool for testing Fortran 77 programs. Given a program and a test set, Mothra generates a set of mutants, executes them against test cases in the test set, and computes the mutation score. ATAC is a data flow coverage measurement tool for $\mathrm{C}$ programs. Given a program and a test set, ATAC computes the block, decision, c-use, p-use, and all-uses coverage. While using these tools, it is the tester's responsibility to determine equivalent mutants and infeasible data flow attributes.

Thirty adequate test sets with respect to mutation, abs/ror mutation, $10 \%$ mutation, and all-uses adequacy criteria were randomly generated with the exception that there are only five $10 \%$ mutation adequate test sets for FIND, STRMAT1, STRMAT2, and TEXTFMT. Details of test case generation can be found in [13]. 
Table 1: Program size metrics

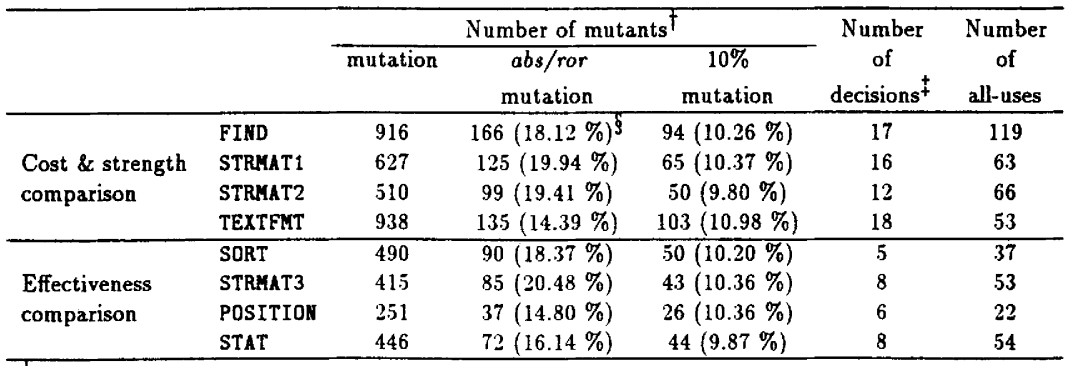

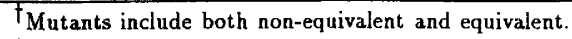

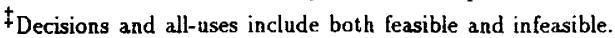

$\oint_{\%}=\frac{\text { number of mutants examined using alternate mutation }}{\text { number of mutants examined in mutation }} * 100$

Table 2: Average size of mutation, $10 \%$ mutation, abs/ror mutation, and all-uses adequate test sets

\begin{tabular}{lcccc}
\hline & FIND & STRMAT1 & STRMAT2 & TEXTFMT \\
\hline mutation & 24.57 & 20.23 & 17.57 & 16.67 \\
10\% mutation & 9.20 & 10.00 & 8.60 & 9.60 \\
abs/ror mutation & 14.73 & 12.00 & 8.27 & 6.97 \\
all-uses & 6.67 & 6.07 & 6.40 & 5.23 \\
\hline
\end{tabular}

\section{Experimental results and analysis}

Table 1 lists the number of mutants, decisions and all-uses for the programs used. These metrics serve as indicators of the relative complexity of programs considered in our experiments. Table 2 lists the average size of mutation, $10 \%$ mutation, abs/ror mutation, and all-uses adequate tests, respectively. Tables 3 and 4 contain the average all-uses and mutation scores using mutation, $10 \%$ mutation, abs/ror mutation, and all-uses adequate test sets, respectively. Scores in the above tables are computed as percentages. Table 5 contains statistics on fault-revealing adequate test sets with respect to mutation, $10 \%$ mutation, abs/ror mutation, and all-uses criteria.

\section{Cost comparison}

From our experimental data and the summary in Table 2 we make the following observations:

- $42 \%$ to $63 \%$ and $40 \%$ to $58 \%$ fewer test cases are required to satisfy $10 \%$ mutation and $a b s /$ ror mutation adequacy, respectively, than to satisfy mutation adequacy.

- $35 \%$ to $84 \%$ and $33 \%$ to $121 \%$ more test cases are required to satisfy $10 \%$ mutation and $a b s /$ ror mutation adequacy, respectively, than to satisfy all-uses adequacy.

- $175 \%$ to $268 \%$ more test cases are required to satisfy mutation adequacy than to satisfy all-uses adequacy.

- $89 \%$ to $90 \%$ and $80 \%$ to $85 \%$ cost reduction in terms of number of mutants to be examined for $10 \%$ mutation and abs/ror mutation adequacy, respectively. 
Table 3: Average all-uses scores using mutation, $10 \%$ mutation, abs/ror mutation, and all-uses adequate test sets

\begin{tabular}{lcccc}
\hline & FIHD & STRHAT1 & STRMAT2 & TEXTFMT \\
\hline mutation & 100.00 & 100.00 & 100.00 & 99.39 \\
10\% mutation & 99.59 & 97.93 & 98.62 & 96.97 \\
abs/ror mutation & 99.56 & 97.13 & 96.27 & 95.45 \\
all-uses & 100.00 & 100.00 & 100.00 & 100.00 \\
\hline
\end{tabular}

Table 4: Average mutation scores using mutation, $10 \%$ mutation, abs/ror mutation, and all-uses adequate test sets

\begin{tabular}{lcccc}
\hline & FIND & STRMAT1 & STRMAT2 & TEXTFIT \\
\hline mutation & 100.00 & 100.00 & 100.00 & 100.00 \\
$10 \%$ mutation & 97.59 & 97.25 & 96.37 & 99.01 \\
abs/ror mutation & 98.29 & 98.84 & 95.70 & 95.90 \\
all-uses & 92.31 & 86.01 & 91.60 & 92.86 \\
\hline
\end{tabular}

\section{Strength comparison}

From our experimental data and the summary in Tables 3 and 4 we make the following observations:

(1) All-uses scores:

- For FIND: Each mutation, three of the five $10 \%$ mutation, and seventeen of the thirty $a b s /$ ror mutation adequate test sets are all-uses adequate.

- For STRMAT1: Each mutation, two of the five $10 \%$ mutation, and eighteen of the thirty $a b s /$ ror mutation adequate test sets are all-uses adequate.

- For STRMAT2: Each mutation, three of the five $10 \%$ mutation, and six of the thirty $a b s /$ ror mutation adequate test sets are all-uses adequate.

- For TEXTFMT: Twenty-four of the thirty mutation, one of the five $10 \%$ mutation, and two of the thirty abs/ror mutation adequate test sets are all-uses adequate.

(2) Mutation scores:

- None of the $10 \%$ mutation, abs/ror mutation, and all-uses adequate test set is mutation adequate.

In summary, $10 \%$ and abs/ror mutation adequate test sets suffer less than $5 \%$ loss on average mutation and all-uses scores when compared with the mutation and all-uses adequate test sets, respectively. Notice that when compared with the all-uses adequacy, both $10 \%$ and abs/ror mutation provide higher mutation scores.

\section{Effectiveness comparison}

From our experimental data and the summary in Table 5, we make the following observations:

- For all four experiments, the mutation criterion is the most effective in exposing program faults.

- The average effectiveness for the mutation, abs/ror mutation, $10 \%$ mutation and all-uses criteria is $92.50 \%, 88.33 \%, 81.66 \%$, and $83.33 \%$, respectively.

The above observations indicate that in our study mutation criterion is the most effective followed, in order, by abs/ror mutation, all-uses and $10 \%$ mutation. 
Table 5: Number of fault-revealing adequate test sets

\begin{tabular}{lcccc}
\hline Program & mutation & abs/ror mutation & $10 \%$ mutation & All-uses \\
\hline SORT & $30(100.00 \%)^{+}$ & $30(100.00 \%)$ & $29(96.67 \%)$ & $29(96.67 \%)$ \\
STRHAT3 & $21(70.00 \%)$ & $16(53.33 \%)$ & $18(60.00 \%)$ & $12(40.00 \%)$ \\
POSITION & $30(100.00 \%)$ & $30(100.00 \%)$ & $28(93.33 \%)$ & $30(100.00 \%)$ \\
STAT & $30(100.00 \%)$ & $30(100.00 \%)$ & $23(76.67 \%)$ & $29(96.67 \%)$ \\
\hline
\end{tabular}

† The percentage was computed using Equation (5) in Section 3.

\section{Conclusions and future work}

In this study we find that compared to the mutation criterion, both the randomly selected $10 \%$ mutation and the constrained abs/ror mutation provide significant cost reductions in terms of the number of test cases required and the number of mutants examined. This gain is, however, accompanied by a small strength loss in the ability to distinguish non-equivalent mutants and cover feasible all-uses. On the other hand, when compared to the all-uses criterion both alternate mutation criteria require more test cases and suffer a small strength loss in the ability to cover feasible all-uses. However, both of them also show a better effectiveness and a stronger strength in the ability to distinguish non-equivalent mutants than the all-uses criterion. Our overall conclusions are:

- In terms of decreasing order of cost (the number of test cases required) the ranking is mutation, abs/ror mutation, $10 \%$ mutation, and all-uses.

- In terms of decreasing order of fault detection effectiveness the ranking is mutation, abs/ror mutation, all-uses, and $10 \%$ mutation.

These data suggest that examining only a small percentage of the mutants may be a useful heuristic for evaluating and constructing test sets in practice. While testing software under time pressure and budget constraints, testers may use mutation testing for the safety-critical parts and leave the other parts to be tested by a cost-effective alternative such as the abs/ror mutation or the all-uses criteria.

As discussed in Section 4.2, the tools we used support different programming languages, Mothra for Fortran 77 and ATAC for C. Although it has been shown that the effect of using different languages is negligible on the conclusions reported here [10], it is certainly desirable to avoid any language translation in the experiments. We are currently using PROTEUM [6], a $\mathrm{C}$ mutation tool implemented based on [2], to conduct experiments with constrained mutation using different combinations of mutant operators directly on larger size $\mathrm{C}$ programs. Such studies have the advantage of eliminating any possible language bias and will shed more light on how to reduce the cost of mutation testing.

\section{References}

1 Acree, T. A.: 'On mutation', PhD thesis, School of Information and Computer Science, Georgia Institute of Technology, Atlanta, GA, August 1980.

2 Agrawal, H., DeMillo, R. A., Hathaway, R., Hsu, Wm., Hsu, W., Krauser, E. W., Martin, R. J., Mathur, A. P. and Spafford, E. H.: 'Design of mutant operators for the C programming language', Technical Report SERC-TR-41-P, Software Engineering Research Center, Purdue University, W. Lafayette, IN, March 1989.

3 Budd, T. A.: 'Mutation Analysis of Program Test Data', PhD thesis, Yale University, New Haven, CT, May 1980. 
4 Choi, B. J., DeMillo, R. A., Krauser, E. W., Mathur, A. P., Martin, R. J., Offutt, A. J., Pan, H. and Spafford, E. H.: 'The Mothra toolset', Proc. of the Twenty-Second Annual Hawaii International Conference on System Sciences, HI, January 1989.

5 Clarke, L. A., Podgurski, A., Richardson, D. J. and Zeil S. J.: 'A formal evaluation of data flow path selection criteria', IEEE Trans. on Software Engineering, 15(11):1318-1332, November 1989.

6 Delamaro, M. E., Maldonado, J. C., Jino, M. and Chaim, M.: 'PROTEUM: A testing tool based on mutation analysis', Proc. of the Seventh Brazilian Symposium on Software Engineering, Rio de Janeiro, Brazil, October 1993.

7 DeMillo, R. A., Lipton, R. J. and Sayward, F. G.: 'Hints on test data selection: Help for the practicing programmer', IEEE Computer, 11(4):34-41, April 1978.

8 Horgan, J. R. and London, S. A.: 'ATAC: A data flow coverage testing tool for C', Proc. of Symposium on Assessment of Quality Software Development Tools, New Orleans, LA, May 1992, pp 2-10

9 Krauser, E. W., Mathur, A. P. and Rego, V. J.: 'High performance software testing on SIMD machines', IEEE Trans. on Software Engineering, 17(5):403-423, May 1991.

10 Mathur, A. P. and Wong, W. E.: 'An empirical comparison of data flow and mutation based test adequacy criteria', The Journal of Software Testing, Verification, and Reliability, 4(1):9-31, March 1994.

11 Weiss, S. N. and Fleyshgakker, V. N.: 'Improved serial algorithms for mutation analysis', Proc. of International Symposium on Software Testing and Analysis, Cambridge, MA, June 1993 , pp 149-158

12 Weyuker, E. J., Weiss, S. N. and Hamlet, R. G.: 'Comparison of program testing strategies', Proc. of the Fourth Symposium on Software Testing, Analysis and Verification, Victoria, British Columbia, Canada, October 1991, pp 154-164

13 Wong, W. E.: 'On Mutation and Data Flow', PhD thesis, Department of Computer Science, Purdue University, W. Lafayette, IN, December 1993.

14 Wong, W. E. and Mathur, A. P.: 'Reducing the cost of mutation testing: An empirical study', The Journal of Systems and Software, 1995. (To appear) 